\title{
AdDENDUM
}

\section{Addendum for Euro Surveill. 2017;22(8)}

\section{Eurosurveillance editorial team ${ }^{1}$}

1. European Centre for Disease Prevention and Control (ECDC), Stockholm, Sweden

2. Anti-infectives and Vaccines Office, European Medicines Agency (EMA), London, United Kingdom

\section{Correspondence: Eurosurveillance editorial team (eurosurveillance@ecdc.europa.eu)}

Citation style for this article:

Eurosurveillance editorial team. Addendum for Euro Surveill. 2017;22(8). Euro Surveill. 2017;22(40):pii=171005. https://doi.org/10.2807/1560-7917. ES.2017.22.40.171005

To the article entitled 'Emergence of a novel subclade of influenza $\mathrm{A}\left(\mathrm{H}_{3} \mathrm{~N}_{2}\right)$ virus in London, December 2016 to January 2017' by H Harvala et al., published on 23 February 2017, the following information is added at the request of the authors.
We gratefully acknowledge the authors, originating and submitting laboratories of the sequences from GISAID's EpiFlu Database on which this research is based. The list is detailed in the Table below.

\section{TABLE A}

Information on GISAID sequences on which the study is based

\begin{tabular}{|c|c|c|c|c|c|}
\hline Virus isolate & $\begin{array}{l}\text { Segment ID/ } \\
\text { Accession } \\
\text { number }\end{array}$ & Country & $\begin{array}{l}\text { Collection } \\
\text { date } \\
\text { (year-month- } \\
\text { day) }\end{array}$ & Originating laboratory & Submitting laboratory \\
\hline A/Stockholm/18/2011 & EPI326139 & Sweden & $2011-03-28$ & $\begin{array}{l}\text { Public Health Agency of } \\
\text { Sweden, Solna, Sweden }\end{array}$ & $\begin{array}{l}\text { National Institute for } \\
\text { Medical Research, } \\
\text { London, UK }\end{array}$ \\
\hline A/Perth/16/2009 & EPI182941 & Australia & 2009-04-07 & $\begin{array}{c}\text { Pathwest QE II Medical } \\
\text { Centre, Nedlands, } \\
\text { Australia }\end{array}$ & $\begin{array}{c}\text { WHO Collaborating Centre } \\
\text { for Reference and Research } \\
\text { on Influenza, Victoria, } \\
\text { Australia }\end{array}$ \\
\hline A/AthensGR/112/2012 & $\mathrm{EPI}_{35} 8885$ & Greece & 2012-02-01 & $\begin{array}{l}\text { Hellenic Pasteur } \\
\text { Institute, } \\
\text { Athens, Greece }\end{array}$ & $\begin{array}{c}\text { National Institute for } \\
\text { Medical Research, } \\
\text { London, UK }\end{array}$ \\
\hline A/Texas/50/2012 & $\mathrm{EPI} 391247$ & United States & $2012-04-15$ & $\begin{array}{c}\text { Texas Department of } \\
\text { State Health Services, } \\
\text { Austin, USA }\end{array}$ & $\begin{array}{c}\text { Centers for Disease } \\
\text { Control and Prevention, } \\
\text { Atlanta, US }\end{array}$ \\
\hline A/Samara/73/2013 & $\mathrm{EPI}_{4} 60558$ & $\begin{array}{l}\text { Russian } \\
\text { Federation }\end{array}$ & $2013-03-12$ & $\begin{array}{c}\text { WHO National } \\
\text { Influenza Centre, } \\
\text { Saint Petersburg, } \\
\text { Russian Federation }\end{array}$ & $\begin{array}{l}\text { National Institute for } \\
\text { Medical Research, } \\
\text { London, UK }\end{array}$ \\
\hline A/Netherlands/525/2014 & $\mathrm{EPI}_{574644}$ & Netherlands & $2014-12-17$ & $\begin{array}{l}\text { National Institute for } \\
\text { Public Health and the } \\
\text { Environment (RIVM), } \\
\text { Bilthoven, Netherlands }\end{array}$ & $\begin{array}{l}\text { National Institute for } \\
\text { Medical Research, } \\
\text { London, UK }\end{array}$ \\
\hline
\end{tabular}

GISAID: Global Initiative on Sharing All Influenza Data; SAR: Special Administrative Regions of the People's Republic of China; UK: United Kingdom; US: United States; WHO: World Health Organization.

All submitters of data may be contacted directly via the GISAID website www.gisaid.org. 


\section{TABLE B}

Information on GISAID sequences on which the study is based

\begin{tabular}{|c|c|c|c|c|c|}
\hline Virus isolate & $\begin{array}{l}\text { Segment ID/ } \\
\text { Accession } \\
\text { number }\end{array}$ & Country & $\begin{array}{l}\text { Collection } \\
\text { date } \\
\text { (year-month- } \\
\text { day) }\end{array}$ & Originating laboratory & Submitting laboratory \\
\hline A/Stockholm/6/2014 & $\mathrm{EPI}_{530677}$ & Sweden & $2014-02-06$ & $\begin{array}{l}\text { Public Health Agency of } \\
\text { Sweden, Solna, Sweden }\end{array}$ & $\begin{array}{l}\text { National Institute for } \\
\text { Medical Research, } \\
\text { London, UK }\end{array}$ \\
\hline A/Glasgow/401678/2015 & $\mathrm{EPI}_{574607}$ & $\begin{array}{l}\text { United } \\
\text { Kingdom }\end{array}$ & 2015-01-07 & $\begin{array}{l}\text { Gart Naval General } \\
\text { Hospital, Glasgow, } \\
\text { United Kingdom }\end{array}$ & $\begin{array}{l}\text { National Institute for } \\
\text { Medical Research, } \\
\text { London, UK }\end{array}$ \\
\hline A/Switzerland/9715293/2013 & $\mathrm{EPI}_{530687}$ & Switzerland & $2013-12-06$ & $\begin{array}{l}\text { Hopital Cantonal } \\
\text { Universitaire de } \\
\text { Geneves, Geneve, } \\
\text { Switzerland }\end{array}$ & $\begin{array}{l}\text { National Institute for } \\
\text { Medical Research, } \\
\text { London, UK }\end{array}$ \\
\hline A/Bucuresti/193605/2016 & $\mathrm{EPI} 769485$ & Romania & 2016-03-08 & $\begin{array}{c}\text { Cantacuzino Institute, } \\
\text { Bucharest, Romania }\end{array}$ & $\begin{array}{l}\text { Crick Worldwide Influenza } \\
\text { Centre, London, UK }\end{array}$ \\
\hline A/Hong Kong/146/2013 & $\mathrm{EPI}_{426061}$ & $\begin{array}{l}\text { Hong Kong } \\
\text { (SAR) }\end{array}$ & 2013-01-11 & $\begin{array}{c}\text { Government Virus Unit, } \\
\text { Kowloon, Hong Kong } \\
\text { (SAR) }\end{array}$ & $\begin{array}{l}\text { National Institute for } \\
\text { Medical Research, } \\
\text { London, UK }\end{array}$ \\
\hline A/Hong Kong/4801/2014 & EPI834581 & $\begin{array}{l}\text { Hong Kong } \\
\text { (SAR) }\end{array}$ & $2014-02-26$ & $\begin{array}{c}\text { National Institute for } \\
\text { Medical Research, } \\
\text { London, UK }\end{array}$ & $\begin{array}{c}\text { Centers for Disease } \\
\text { Control and Prevention, } \\
\text { Atlanta, US }\end{array}$ \\
\hline A/Hong Kong/5738/2014 & $\mathrm{EPI}_{539806}$ & $\begin{array}{l}\text { Hong Kong } \\
\text { (SAR) }\end{array}$ & 2014-04-30 & $\begin{array}{c}\text { Government Virus Unit, } \\
\text { Kowloon, Hong Kong } \\
\text { (SAR) }\end{array}$ & $\begin{array}{l}\text { National Institute for } \\
\text { Medical Research, } \\
\text { London, UK }\end{array}$ \\
\hline A/Glasgow/400580/2014 & $\mathrm{EPI}_{574601}$ & $\begin{array}{l}\text { United } \\
\text { Kingdom }\end{array}$ & $2014-12-26$ & $\begin{array}{l}\text { Gart Naval General } \\
\text { Hospital, Glasgow, } \\
\text { United Kingdom }\end{array}$ & $\begin{array}{l}\text { National Institute for } \\
\text { Medical Research, } \\
\text { London, UK }\end{array}$ \\
\hline A/South Africa/vwo071/2016 & EPI829363 & South Africa & $2016-07-21$ & $\begin{array}{c}\text { National Institute } \\
\text { for Communicable } \\
\text { Diseases, Sandringham, } \\
\text { South Africa }\end{array}$ & $\begin{array}{l}\text { Crick Worldwide Influenza } \\
\text { Centre, London, UK }\end{array}$ \\
\hline A/Cote Dlvoire/651/2016 & $\mathrm{EPI} 781608$ & Cote d'Ivoire & $2016-04-25$ & $\begin{array}{l}\text { Pasteur Institut of Côte } \\
\text { d'Ivoire, } \\
\text { Abidjan, Cote d'Ivoire }\end{array}$ & $\begin{array}{l}\text { Crick Worldwide Influenza } \\
\text { Centre, London, UK }\end{array}$ \\
\hline A/Cote Dlvoire/697/2016 & $\mathrm{EPI} 781616$ & Cote d'Ivoire & $2016-05-04$ & $\begin{array}{l}\text { Pasteur Institut of Côte } \\
\text { d‘Ivoire } \\
\text { Abidjan, Cote d'Ivoire }\end{array}$ & $\begin{array}{l}\text { Crick Worldwide Influenza } \\
\text { Centre, London, UK }\end{array}$ \\
\hline A/Cote Dlvoire/599/2016 & $\mathrm{EPI} 781602$ & Cote d'Ivoire & 2016-04-15 & $\begin{array}{l}\text { Pasteur Institut of Côte } \\
\text { d'Ivoire } \\
\text { Abidjan, Cote d'Ivoire }\end{array}$ & $\begin{array}{l}\text { Crick Worldwide Influenza } \\
\text { Centre, London, UK }\end{array}$ \\
\hline A/Ghana/FS-16-0889/2016 & EPI829309 & Ghana & $2016-06-26$ & $\begin{array}{c}\text { Noguchi Memorial } \\
\text { Institute for Medical } \\
\text { Research, Accra, Ghana }\end{array}$ & $\begin{array}{l}\text { Crick Worldwide Influenza } \\
\text { Centre, London, UK }\end{array}$ \\
\hline A/Ghana/FS-16-0695/2016 & EPI829307 & Ghana & $2016-05-23$ & $\begin{array}{c}\text { Noguchi Memorial } \\
\text { Institute for Medical } \\
\text { Research, Accra, Ghana }\end{array}$ & $\begin{array}{l}\text { Crick Worldwide Influenza } \\
\text { Centre, London, UK }\end{array}$ \\
\hline
\end{tabular}

GISAID: Global Initiative on Sharing All Influenza Data; SAR: Special Administrative Regions of the People's Republic of China; UK: United Kingdom; US: United States; WHO: World Health Organization.

All submitters of data may be contacted directly via the GISAID website www.gisaid.org. 
TABLE C

Information on GISAID sequences on which the study is based

\begin{tabular}{|c|c|c|c|c|c|}
\hline Virus isolate & $\begin{array}{l}\text { Segment ID/ } \\
\text { Accession } \\
\text { number }\end{array}$ & Country & $\begin{array}{l}\text { Collection } \\
\text { date } \\
\text { (year-month- } \\
\text { day) }\end{array}$ & Originating laboratory & Submitting laboratory \\
\hline A/Ghana/DILI-16-0661/2016 & EPI829305 & Ghana & $2016-06-27$ & $\begin{array}{c}\text { Noguchi Memorial } \\
\text { Institute for Medical } \\
\text { Research, Accra, Ghana }\end{array}$ & $\begin{array}{l}\text { Crick Worldwide Influenza } \\
\text { Centre, London, UK }\end{array}$ \\
\hline A/Stockholm/24/2016 & $\mathrm{EPI}_{781630}$ & Sweden & $2016-02-22$ & $\begin{array}{l}\text { Public Health Agency of } \\
\text { Sweden, Solna, Sweden }\end{array}$ & $\begin{array}{l}\text { Crick Worldwide Influenza } \\
\text { Centre, London, UK }\end{array}$ \\
\hline A/Oman/1807/2016 & EPI769519 & Oman & $2016-02-12$ & $\begin{array}{c}\text { Central Public Health } \\
\text { Laboratory, Muscat, } \\
\text { Oman } \\
\end{array}$ & $\begin{array}{l}\text { Crick Worldwide Influenza } \\
\text { Centre, London, UK }\end{array}$ \\
\hline A/Glasgow/431929/2014 & $\mathrm{EPI}_{574704}$ & $\begin{array}{l}\text { United } \\
\text { Kingdom }\end{array}$ & $2014-11-23$ & $\begin{array}{l}\text { Gart Naval General } \\
\text { Hospital, Glasgow, } \\
\text { United Kingdom }\end{array}$ & $\begin{array}{l}\text { National Institute for } \\
\text { Medical Research, } \\
\text { London, UK }\end{array}$ \\
\hline A/Glasgow/400097/2015 & EPI574599 & $\begin{array}{l}\text { United } \\
\text { Kingdom }\end{array}$ & 2015-01-02 & $\begin{array}{l}\text { Gart Naval General } \\
\text { Hospital, Glasgow, } \\
\text { United Kingdom }\end{array}$ & $\begin{array}{c}\text { National Institute for } \\
\text { Medical Research, } \\
\text { London, UK }\end{array}$ \\
\hline A/Glasgow/401674/2015 & $\mathrm{EPI}_{574605}$ & $\begin{array}{l}\text { United } \\
\text { Kingdom }\end{array}$ & 2015-01-01 & $\begin{array}{l}\text { Gart Naval General } \\
\text { Hospital, Glasgow, } \\
\text { United Kingdom }\end{array}$ & $\begin{array}{l}\text { National Institute for } \\
\text { Medical Research, } \\
\text { London, UK }\end{array}$ \\
\hline A/Glasgow/401673/2014 & $\mathrm{EPI}_{574603}$ & $\begin{array}{l}\text { United } \\
\text { Kingdom }\end{array}$ & $2014-12-29$ & $\begin{array}{l}\text { Gart Naval General } \\
\text { Hospital, Glasgow, } \\
\text { United Kingdom }\end{array}$ & $\begin{array}{l}\text { National Institute for } \\
\text { Medical Research, } \\
\text { London, UK }\end{array}$ \\
\hline A/Serbia-NS/1517/2016 & EPI746057 & Serbia & $2016-02-10$ & $\begin{array}{l}\text { I Institute of Public } \\
\text { Health, Novi Sad, } \\
\text { Serbia }\end{array}$ & $\begin{array}{l}\text { Crick Worldwide Influenza } \\
\text { Centre, London, UK }\end{array}$ \\
\hline A/South Africa/R2665/2015 & EPI630781 & South Africa & $2015-05-25$ & $\begin{array}{c}\text { National Institute } \\
\text { for Communicable } \\
\text { Diseases, Sandringham, } \\
\text { South Africa }\end{array}$ & $\begin{array}{l}\text { Crick Worldwide Influenza } \\
\text { Centre, London, UK }\end{array}$ \\
\hline A/Georgia/532/2015 & $\mathrm{EPI}_{5} 89699$ & Georgia & 2015-03-09 & $\begin{array}{c}\text { National Centre for } \\
\text { Disease Control and } \\
\text { Public Health, Tbilisi, } \\
\text { Georgia }\end{array}$ & $\begin{array}{l}\text { Crick Worldwide Influenza } \\
\text { Centre, London, UK }\end{array}$ \\
\hline A/Glasgow/400003/2015 & $\mathrm{EPI}_{574597}$ & $\begin{array}{l}\text { United } \\
\text { Kingdom }\end{array}$ & 2015-01-02 & $\begin{array}{l}\text { Gart Naval General } \\
\text { Hospital, Glasgow, } \\
\text { United Kingdom }\end{array}$ & $\begin{array}{l}\text { National Institute for } \\
\text { Medical Research, } \\
\text { London, UK }\end{array}$ \\
\hline A/Hong Kong/7295/2014 & $\mathrm{EPI}_{551882}$ & $\begin{array}{l}\text { Hong Kong } \\
\text { (SAR) }\end{array}$ & 2014-08-07 & $\begin{array}{c}\text { Government Virus Unit, } \\
\text { Kowloon, Hong Kong } \\
\text { (SAR) }\end{array}$ & $\begin{array}{l}\text { National Institute for } \\
\text { Medical Research, } \\
\text { London, UK }\end{array}$ \\
\hline A/Slovenia/3188/2015 & EPI699750 & Slovenia & $2015-12-26$ & $\begin{array}{c}\text { Laboratory for Virology, } \\
\text { National Institute } \\
\text { of Public Health, } \\
\text { Ljubljana, Slovenia }\end{array}$ & $\begin{array}{l}\text { Crick Worldwide Influenza } \\
\text { Centre, London, UK }\end{array}$ \\
\hline A/Victoria/522/2016 & EPI831759 & Australia & 2016-07-04 & $\begin{array}{c}\text { Monash Medical Centre, } \\
\text { Victoria, Australia }\end{array}$ & $\begin{array}{c}\text { WHO Collaborating Centre } \\
\text { for Reference and Research } \\
\text { on Influenza, } \\
\text { Victoria, Australia }\end{array}$ \\
\hline
\end{tabular}

GISAID: Global Initiative on Sharing All Influenza Data; SAR: Special Administrative Regions of the People's Republic of China; UK: United Kingdom; US: United States; WHO: World Health Organization.

All submitters of data may be contacted directly via the GISAID website www.gisaid.org. 


\section{TABLE D}

Information on GISAID sequences on which the study is based

\begin{tabular}{|c|c|c|c|c|c|}
\hline Virus isolate & $\begin{array}{l}\text { Segment ID/ } \\
\text { Accession } \\
\text { number }\end{array}$ & Country & $\begin{array}{l}\text { Collection } \\
\text { date } \\
\text { (year-month- } \\
\text { day) }\end{array}$ & Originating laboratory & Submitting laboratory \\
\hline A/Victoria/512/2016 & EPI831633 & Australia & $2016-07-13$ & $\begin{array}{l}\text { Monash Medical Centre, } \\
\text { Victoria, Australia }\end{array}$ & $\begin{array}{c}\text { WHO Collaborating Centre } \\
\text { for Reference and Research } \\
\text { on Influenza, } \\
\text { Victoria, Australia }\end{array}$ \\
\hline A/Victoria/545/2016 & EPI831876 & Australia & $2016-07-31$ & $\begin{array}{c}\text { Monash Medical Centre, } \\
\text { Victoria, Australia }\end{array}$ & $\begin{array}{c}\text { WHO Collaborating Centre } \\
\text { for Reference and Research } \\
\text { on Influenza, } \\
\text { Victoria, Australia }\end{array}$ \\
\hline
\end{tabular}

GISAID: Global Initiative on Sharing All Influenza Data; SAR: Special Administrative Regions of the People’s Republic of China; UK: United Kingdom; US: United States; WHO: World Health Organization.

All submitters of data may be contacted directly via the GISAID website www.gisaid.org.

\section{License and copyright}

This is an open-access article distributed under the terms of the Creative Commons Attribution (CC BY 4.0) Licence. You may share and adapt the material, but must give appropriate credit to the source, provide a link to the licence, and indicate if changes were made.

This article is copyright of the European Centre for Disease Prevention and Control, 2017. 